\title{
Experimental and Theoretical Studies on the Structure and Photoluminescent Properties of New Mononuclear and Homodinuclear Europium(III) $\beta$-Diketonate Complexes
}

\author{
João P. Martins, ${ }^{1}$ Pablo Martín-Ramos, ${ }^{1,2}$ \\ Pedro Chamorro-Posada, ${ }^{3}$ Pedro S. Pereira Silva, ${ }^{1}$ Jesús Martín-Gil, ${ }^{4}$ \\ Salvador Hernández-Navarro, ${ }^{4}$ and Manuela Ramos Silva ${ }^{1}$ \\ ${ }^{1}$ CFisUC, Department of Physics, University of Coimbra, Rua Larga, 3004-516 Coimbra, Portugal \\ ${ }^{2}$ Higher Polytechnic School of Huesca, University of Zaragoza, Carretera de Cuarte, s/n, 22071 Huesca, Spain \\ ${ }^{3}$ Signal Theory, Communications and Telematics Engineering Department, ETSIT, University of Valladolid, \\ Campus Miguel Delibes, Paseo Belén 15, 47011 Valladolid, Spain \\ ${ }^{4}$ Advanced Materials Laboratory, ETSIIAA, University of Valladolid, Avenida de Madrid 44, 34004 Palencia, Spain
}

Correspondence should be addressed to Manuela Ramos Silva; manuela@pollux.fis.uc.pt

Received 19 April 2015; Accepted 10 June 2015

Academic Editor: Jörg Fink

Copyright (C) 2015 João P. Martins et al. This is an open access article distributed under the Creative Commons Attribution License, which permits unrestricted use, distribution, and reproduction in any medium, provided the original work is properly cited.

\begin{abstract}
Two novel europium(III) complexes, a monomer and a homodimer, with 1-(4-chlorophenyl)-4,4,4-trifluoro-1,3-butanedione (Hcbtfa) and 5-chloro-1,10-phenanthroline (cphen) ligands, formulated as [Eu(cbtfa $\left.)_{3}(\mathrm{cphen})\right]$ and $\left[\mathrm{Eu}_{2}(\mathrm{cbtfa})_{4}(\mathrm{cphen})_{2}\left(\mathrm{CH}_{3} \mathrm{O}\right)_{2}\right]$, have been synthesized. Their structures have been elucidated by X-ray diffraction and their absorption and emission properties have been studied in the solid state. The experimental data has then been used to test the recently released LUMPAC software, a promising tool which can facilitate the design of more efficient lanthanide light-conversion molecular devices by combining ground state geometry, excited state energy, and luminescent properties calculations.
\end{abstract}

\section{Introduction}

The development of strategies for the design of highly luminescent lanthanide coordination complexes is of crucial importance with a view to a wide range of technological applications, which encompass, for example, organic-light emitting diodes (OLEDs), luminescent probes in biomedical assays, time resolved microscopy, luminescent sensors for chemical species, or coatings for photovoltaics $[1,2]$. Such strategies, aimed at optimizing the quantum yields and other relevant properties, ultimately depend on gaining a better insight on the correlation of those properties with structural data, and the combination of experimental and theoretical characterization resources is deemed as a very promising approach.

In the particular case of Eu(III)-based light-conversion molecular devices (LCMDs), the application of theoretical chemistry methods to analyse the changes in structural parameters, bond energies, and other properties of the lanthanide complexes as a function of ligand types - in order to boost their luminescence quantum yield-has been a recurrent theme in the literature, from the pioneering work of de Sá et al. back to 2000 [3] to the work of Freire et al. [46 ] or to the more recent and comprehensive approaches of, for example, Lima et al. [7].

Amongst the different theoretical models available, the semiempirical Sparkle [6] model is particularly attractive for the determination of ground state geometries, since it can attain a similar accuracy to that achieved by $a b$ initio/ECP full geometry optimization calculations with significantly lower CPU times [8-10]. However, the prediction of luminescent properties (such as singlet and triplet energy states, intensity parameters, energy transfer and back-transfer rates, radiative and nonradiative rates, and quantum efficiencies or quantum 


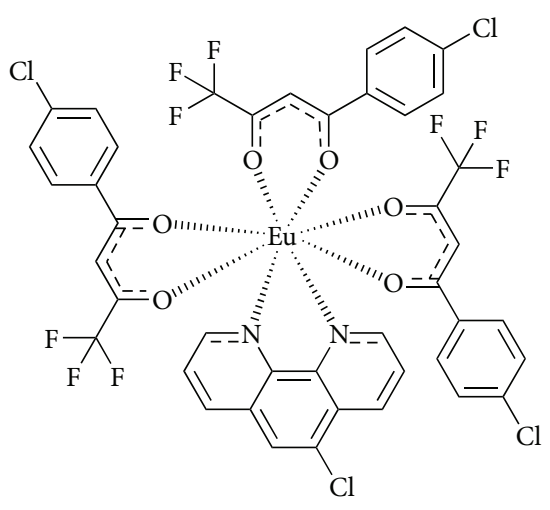

(a)

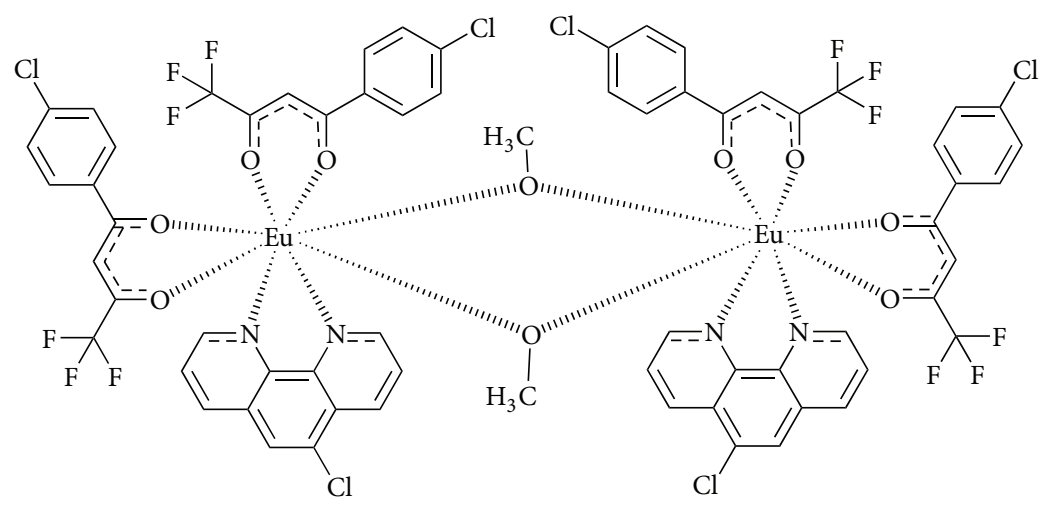

(b)

Figure 1: Chemical structures of complex 1 (a) and complex 2 (b).

yields) from the optimized ground state geometries could be regarded as an unresolved matter due to the lack of convenient software tools. This situation has recently changed due to release of the free and user-friendly LUMPAC software [11], which covers these calculations in its third module.

Encouraged by the first results published by the group who developed the software $[12,13]$, we have hereby assessed LUMPAC for the theoretical study of two closely related $\mathrm{Eu}^{3+}$ complexes, a monomer and a homodinuclear complex, in which the $\mathrm{Eu}^{3+}$ ion(s) is(are) coordinated by the same $\beta$-diketonate (Hcbtfa) and the same diimide (cphen). The chosen $\beta$-diketonate, Hcbtfa, is a more halogenated variant of 4,4,4-trifluoro-1-phenyl-1,3-butanedione (btfa), which has been deemed as one of the best possible choices in terms of maximizing the $\mathrm{Eu}^{3+}$ luminescence quantum yield [7]. In fact, an analogous monomeric complex with Hcbtfa and bathophenanthroline, $\left[\mathrm{Eu}(\mathrm{cbtfa})_{3}\right.$ (bath)] [14], recently attained a quantum efficiency of ca. $60 \%$ and was successfully tested as chromophore for cost-effective OLEDs.

In this study, the synthesis, X-ray structure, and luminescent properties of the aforementioned two novel $\mathrm{Eu}^{3+}$ based materials are reported, and this data has then been used as a reference in order to evaluate the suitability of the semiempirical calculation methods for predicting the equilibrium energy configuration, the electronic properties (resorting to INDO/S-CIS method), and the luminescent properties of the complexes, making use of the different modules of LUMPAC software.

\section{Experimental and Computational Methods}

2.1. Materials and Synthesis. All reagents and solvents employed were commercially available and used without further purification. All the procedures for complex preparation were carried out under nitrogen and using dry reagents to avoid the presence of water and oxygen so as to avoid metal photoluminescence (PL) quenching issues.

Tris(1,4-chlorophenyl-4,4,4-trifluoro-1,3-butanedionate) mono(5-chloro-1,10-phenanthroline) europium(III), complex 1 (Figure $1(\mathrm{a})$ ), was obtained as follows: under stirring, $\mathrm{Eu}\left(\mathrm{NO}_{3}\right)_{3} \cdot 5 \mathrm{H}_{2} \mathrm{O}(1 \mathrm{mmol}, 99.9 \%$ purum, CAS number
63026-01-7, Sigma Aldrich) was mixed with 1,4-chlorophenyl-4,4,4-trifluoro-1,3-butanedione (3 mmol, 97\% purum, CAS number 18931-60-7, Sigma Aldrich) in methanol $(20 \mathrm{~mL})$, and a potassium methylate solution $(3 \mathrm{mmol}, 95 \%$ purum, CAS number 865-33-8, Sigma Aldrich) in methanol was added to neutralize the mixture. $\mathrm{KNO}_{3}$ was removed by decanting, and 5-chloro-1,10-phenanthroline (1 mmol, 98\% purum, CAS number 4199-89-7, Sigma Aldrich) was finally added. The mixture was heated to $75^{\circ} \mathrm{C}$ and stirred overnight, then washed with 1,4-dioxane, and finally dried in vacuum to give the product in $90-95 \%$ yield (based on Eu). Crystals suitable for X-ray analysis were obtained by slow evaporation of a methanol-dioxane solution at room temperature (RT).

Tetra(1,4-chlorophenyl-4,4,4-trifluoro-1,3-butanedionate) $\mathrm{di}$ (5-chloro-1,10-phenanthroline) $\mathrm{di}-\mu_{2}$-methanolate dieuropium(III), complex 2 (Figure $1(\mathrm{~b})$ ), was obtained as a byproduct of aforementioned synthesis procedure.

2.2. X-Ray Crystallographic Analysis. For the determination of the two crystal structures presented in this paper, single crystals were glued to glass fibres and mounted on a Bruker APEX II diffractometer. In both cases, diffraction data was collected at room temperature 293(2) K using graphite monochromated $\mathrm{MoKa}(\lambda=0.71073 \AA)$ radiation. Absorption corrections were made using SADABS [18]. The structures were solved by direct methods using SHELXS97 and refined anisotropically (non-H atoms) by full-matrix least-squares on $F^{2}$ using the SHELXL-97 program [19]. PLATON [20] was used for analyzing the structure and for figure plotting. All the tested crystals of complex 1 were found to be ill-formed and unstable during the few hours of the data collections. To be able to refine a sensible molecular model the whole arsenal of SHELXL restraints had to be used (ISOR, DFIX, FLAT, and SIMU). The final model is just an approximate model good enough to be used as a starting point for the semiempirical methods. Atomic coordinates, thermal parameters, and bond lengths and angles have been deposited at the Cambridge Crystallographic Data Centre (CCDC). Any request to the CCDC for this material should quote the full literature citation and the reference numbers CCDC 1057047-1057048. 


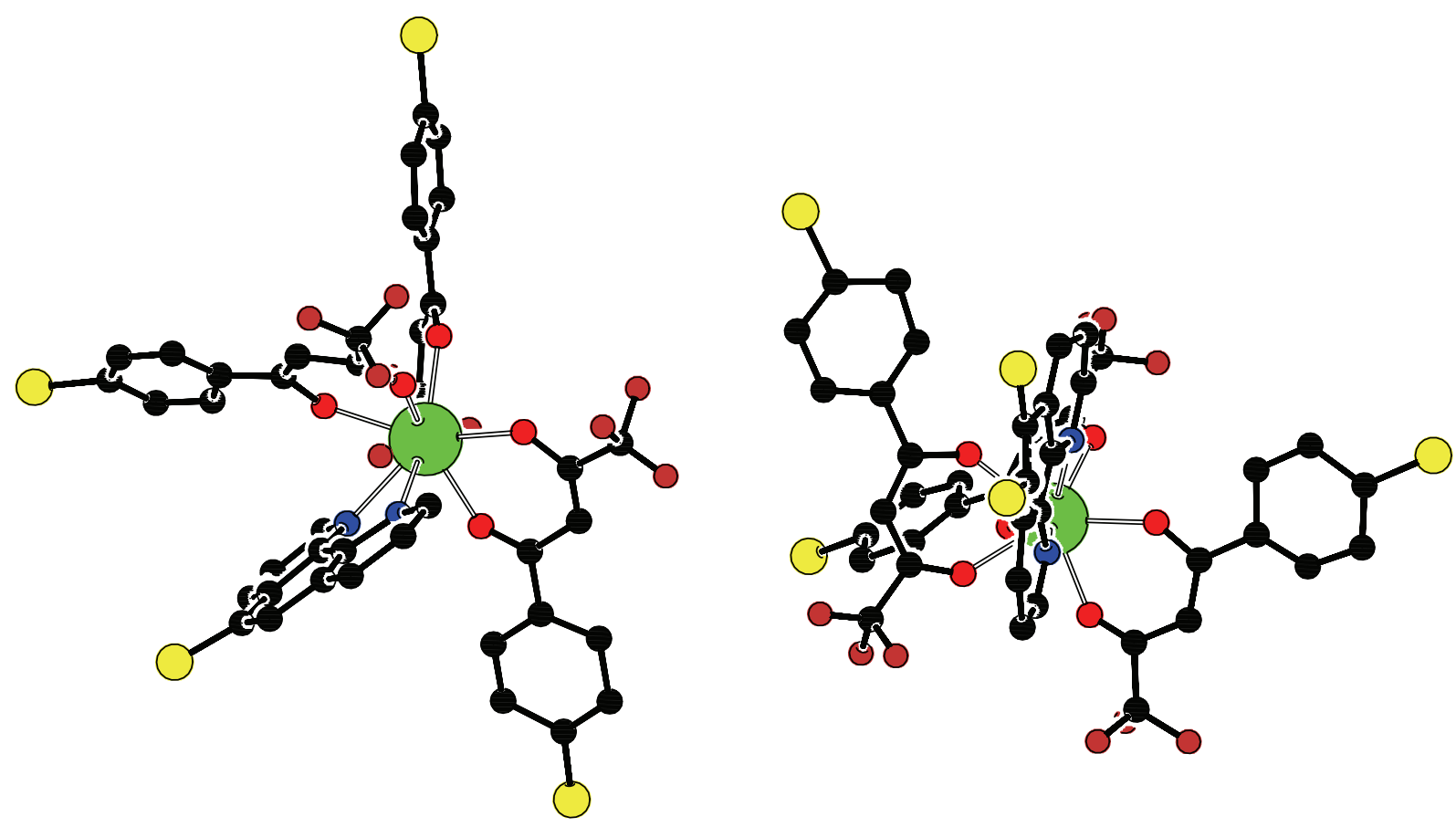

Figure 2: Structural diagram of complex 1, tris(1,4-chlorophenyl-4,4,4-trifluoro-1,3-butanedionate) mono(5-chloro-1,10-phenanthroline) europium(III). $\mathrm{H}$ atoms have been omitted for clarity reasons.

2.3. Spectroscopic Measurements. Optical absorption and photoluminescence spectra of the materials were measured at room temperature. The $285-800 \mathrm{~nm}$ range absorption spectra were recorded with a Cary 4000 Varian spectrophotometer in powder form. Photoluminescence spectra of the materialsin powder-were obtained at room temperature using a modular spectrophotometer Horiba-Jobin-Yvon SPEX Fluorolog 3. All spectra have been corrected by the spectral response of the experimental setups.

2.4. Computational Methods. Using the experimental crystallographic data as an initial guess, the ground state geometries were obtained using the Sparkle/PM6 [21, 22] and Sparkle/PM7 [23, 24] models implemented in the MOPAC2012 software $[25,26]$ using periodic boundary conditions. One unit cell for each of the complexes was employed in the calculations by setting the keyword MERS = $(1,1,1)$. Geometry optimizations were performed for isolated complexes as well. Additionally, the corresponding vibrational frequencies were computed for the PM6 optimized geometries for the two complexes in the gas phase. No imaginary vibrational frequencies were found for any of the geometries, confirming that the results obtained corresponded to true ground states. These computations were performed on a Debian Linux server with four AMD Opteron 16 Core processors and $128 \mathrm{~GB}$ of memory and a Linux operating system.

The electronic spectra for each of the optimized structures were calculated using the ORCA electronic structure package $[27,28]$ via the intermediate neglect of differential overlap/spectroscopic (INDO/S) method and configuration interaction with singles (CIS) [29-31] replacing the $\mathrm{Eu}^{3+}$ ions with point charges as described by de Andrade et al. $[32,33]$. Version 3.0.1 was used for calculations, invoked using LUMPAC 1.0 distribution.

The geometry optimization and the analysis of electronic transitions using the INDO/S-CIS method are integrated in a user-friendly manner in the first and second modules, respectively, of LUMPAC software ecosystem. The third module is a unique feature of LUMPAC and permits calculating the Judd-Ofelt intensity parameters [34] and the estimation of metal-ligand energy transfer and back-transfer rates [35], the radiative and nonradiative emission rates, theoretical quantum efficiency, and emission quantum yield [36]. Calculations within LUMPAC modules were performed using a Windows 8 Toshiba Satellite Core i5 laptop computer.

The theoretical Judd-Ofelt parameters are determined by adjusting the charge factors and polarizabilities to reproduce the experimental values. In this calculation, it is required to provide the coordination polyhedron of the system as input. For the highly symmetric binuclear complex, the analyses have been performed by independently treating the two europium ions with their corresponding coordination polyhedra as in the individual polyhedron method reported by Dutra et al. [13].

\section{Results and Discussion}

3.1. Structural Description. In the mononuclear complex, complex 1 , the $\mathrm{Eu}^{3+}$ ions are coordinated by three negatively charged $\beta$-diketonate ligands and a neutral ancillary $\mathrm{N}, \mathrm{N}$ donor moiety (Figure 2, Table 1). There are two symmetry 
TABLE 1: Crystal data and structure refinement for the mononuclear and the homodinuclear $\mathrm{Eu}^{3+}$ complexes.

\begin{tabular}{|c|c|c|}
\hline Complex & Complex 1 & Complex 2 \\
\hline Empirical formula & $\mathrm{C}_{42} \mathrm{H}_{22} \mathrm{Cl}_{4} \mathrm{EuF}_{9} \mathrm{~N}_{2} \mathrm{O}_{6}$ & $\mathrm{C}_{66} \mathrm{H}_{40} \mathrm{Cl}_{6} \mathrm{Eu}_{2} \mathrm{~F}_{12} \mathrm{~N}_{4} \mathrm{O}_{10}$ \\
\hline Formula weight & 1115.38 & 1793.64 \\
\hline Temperature $(\mathrm{K})$ & $293(2)$ & $293(2)$ \\
\hline Wavelength $(\AA)$ & 0.7107 & 0.7107 \\
\hline Crystal system & Triclinic & Triclinic \\
\hline Space group & P-1 & P-1 \\
\hline$a(\AA)$ & $13.246(5)$ & $10.2575(11)$ \\
\hline$b(\AA)$ & $18.252(7)$ & $12.9224(15)$ \\
\hline$c(\AA)$ & $21.081(8)$ & $15.0618(16)$ \\
\hline$\alpha\left({ }^{\circ}\right)$ & $98.241(8)$ & $72.179(6)$ \\
\hline$\beta\left(^{\circ}\right)$ & $99.461(11)$ & $80.460(6)$ \\
\hline$\gamma\left({ }^{\circ}\right)$ & $108.751(10)$ & $84.813(6)$ \\
\hline Volume $\left(\AA^{3}\right)$ & $4654(3)$ & $1872.7(4)$ \\
\hline$Z$ & 4 & 1 \\
\hline Calculated density $\left(\mathrm{g} \mathrm{cm}^{-3}\right)$ & 1.592 & 1.590 \\
\hline Absorption coefficient $\left(\mathrm{mm}^{-1}\right)$ & 1.659 & 1.958 \\
\hline$F(000)$ & 2192 & 880 \\
\hline$\theta$ range for data collection & $1.67-25.87^{\circ}$ & $1.44-25.80^{\circ}$ \\
\hline Index ranges & $-16<h<10 ;-22<k<18 ;-16<l<25$ & $-11<h<12 ;-15<k<15 ;-16<l<18$ \\
\hline Reflections collected & 20295 & 33633 \\
\hline Independent reflections & 3654 & 4769 \\
\hline Completeness to $2 \theta=51^{\circ}$ & $89 \%$ & $98 \%$ \\
\hline Refinement method & Full-matrix LS on $F^{2}$ & Full-matrix LS on $F^{2}$ \\
\hline Data/restraints/parameters & $16025 / 299 / 1181$ & $7048 / 0 / 452$ \\
\hline Goodness-of-fit on $F^{2}$ & 0.978 & 0.980 \\
\hline Final $R$ indices $[I>2 \sigma(I)]$ & $R=0.1169 ; w R=0.1820$ & $R=0.0436 ; w R=0.0792$ \\
\hline$R$ indices (all data) & $R=0.2894 ; w R=0.2159$ & $R=0.1012 ; w R=0.1097$ \\
\hline Largest difference peak and hole & $-1.801 / 1.623$ & $-0.693 / 1.076$ \\
\hline
\end{tabular}

independent complexes in the unit cell. The coordination spheres of these monomers consist in a square antiprismatic geometry. Table 2 summarizes the Eu-N and Eu-O distances for both complexes in the unit cell, affected by large experimental uncertainties. Nevertheless, all of them are within the normal ranges reported in the literature [17, 37]. Both monomers show heavy disorder, particularly evident for the $\mathrm{CF}_{3}$ groups of the cbtfa ligands and for the $\mathrm{Cl}$ substitute of the phenanthroline. The structure contains large solvent accessible voids.

Complex 2 (Figure 3, Table 1) corresponds to a dimeric variation of complex 1, in which the $\mathrm{Eu}^{3+}$ ions are bridged by two methanolate ions. Each $\mathrm{Eu}^{3+}$ ion is coordinated by two negatively charged $\beta$-diketonate (cbtfa) ligands, a neutral diimide ligand (cphen), and the two bridging methanolate ions. Complex 2 crystallizes in a triclinic centrosymmetric cell with the center of symmetry lying in the middle point of the dimer. There is one dimer per unit cell.

Coordination distances are within the normal ranges reported in the literature $[14,17,37]$, and the same applies to the bite angles, with values close to $70^{\circ}$ for the O...Eu...O angles and of ca. $60^{\circ}$ for the N...Eu...N angle. The coordination sphere corresponds to a square antiprism. The $\mathrm{Eu}^{3+}$ ions are at a distance of $3.742 \AA$ within the dimer.

No conventional $\mathrm{H}$-bonds were found joining the dimers. The packing seems to be influenced by $\pi \ldots \pi$ interactions (Figure 4). Neighboring phenanthroline rings are at $4.143 \AA$ distances (centroid-to-centroid) with a slippage of $2.089 \AA$.

The low crystallinity of the bulk synthesized material, in which the single crystals of complexes 1 and 2 were mixed with more amorphous material, prevented a reliable determination of the proportions of complex 1 and complex 2 by means of X-ray powder diffraction.

3.2. Modeling of the Structures by Semiempirical Methods. A comparison of the unit cell parameters obtained for the PM6 and PM7 predicted structures (e.g., Figure 5) versus those of the SC-XRD data is summarized in Table 3. The percent errors are below 5\% in all cases (generally below 2\%). They are similar for both Hamiltonians in the case of complex 1, while the estimation with PM7 is slightly better in the case of complex 2 for all parameters except for the $c$ value (which in turn leads to an incorrect volume estimation).

The Eu-N and Eu-O distances and some selected angles in the ground state geometries of the monomer and the 


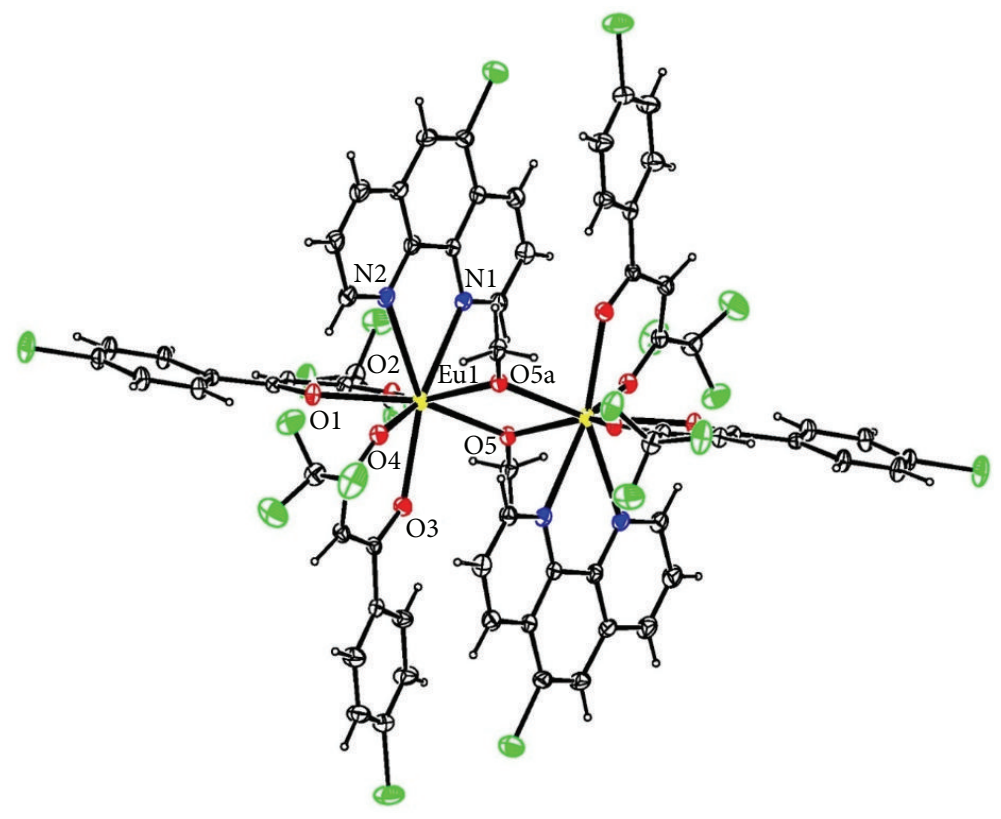

FIGURE 3: Structural diagram of complex 2, namely, tetra(1,4-chlorophenyl-4,4,4-trifluoro-1,3-butanedionate) di(5-chloro-1,10-phenanthroline) di- $\mu_{2}$-methanolate dieuropium(III). $\mathrm{H}$ atoms have been omitted for clarity reasons and displacement ellipsoids were drawn at the $10 \%$ probability level.

TABLE 2: Selected distances and angles ( $\AA$, degrees) for the mononuclear and the homodinuclear $\mathrm{Eu}^{3+}$ complexes.

\begin{tabular}{lccc}
\hline Bond/angle & Complex 1 & Bond/angle & Complex 2 \\
\hline Eu1-O1 & $2.407(3)$ & Eu1-O1 & $2.376(3)$ \\
Eu1-O2 & $2.390(4)$ & Eu1-O2 & $2.284(2)$ \\
Eu1-O3 & $2.393(4)$ & Eu1-O3 & $2.403(3)$ \\
Eu1-O4 & $2.396(4)$ & Eu1-O4 & $2.404(3)$ \\
Eu1-O5 & $2.302(3)$ & Eul-O5 & $2.328(3)$ \\
Eu1-N1 & $2.641(5)$ & Eu1-O6 & $2.389(3)$ \\
Eu1-N2 & $2.646(5)$ & Eu1-N1 & $2.546(4)$ \\
O1-Eu1-O2 & $69.65(12)$ & Eu1-N2 & $2.562(3)$ \\
O3-Eu1-O4 & $71.13(13)$ & Eu2-O7 & $2.339(3)$ \\
N1-Eu1-N2 & $61.73(15)$ & Eu2-O8 & $2.354(2)$ \\
& & Eu2-O9 & $2.208(3)$ \\
& & Eu2-O10 & $2.359(3)$ \\
& & Eu2-O11 & $2.293(3)$ \\
& & Eu2-O12 & $2.356(3)$ \\
& & Eu2-N3 & $2.621(4)$ \\
& & Eu2-N4 & $2.594(3)$ \\
& & O1-Eu1-O2 & $69.69(8)$ \\
& & O3-Eu1-O4 & $70.82(10)$ \\
& & N1-Eu1-N2 & $62.85(12)$ \\
& & O7-Eu2-O8 & $71.62(9)$ \\
& & O9-Eu2-O10 & $70.91(11)$ \\
& & O11-Eu2-O12 & $70.89(8)$ \\
& & $63.74(12)$ \\
\hline & & &
\end{tabular}

dimer (using either the PM6 or the PM7 Hamiltonian with periodic boundary conditions) are compared with those of the actual structures obtained from SC-XRD data in Table 4. The distance values obtained using the PM7 Hamiltonian are remarkably closer to the experimental average values than those attained with the PM6 Hamiltonian in all cases. This is particularly obvious for the Eu-O distances in the dimer: while in the PM6 case they are very similar in all cases (with a significant error for the bridging methanol bonds), PM7 Hamiltonian gives a much more accurate estimation and distinguishes between the bonds associated with cbtfa ligands and those corresponding to bridging methanol molecules. Regarding the angle values, the percent errors are significantly higher than those for the bond lengths, but PM7 errors are consistently lower than those for PM6.

This is in agreement with the observations made by Dutra et al. [24]: a significant increase in accuracy has been achieved in PM7 after relatively minor changes were made to the approximations and after proxy reference data functions representing noncovalent interactions were introduced, leading to a reduction of errors in PM7 geometries by over one-third relative to those of PM6. On the other hand, PM7 method can show worse convergence properties when compared with PM6, as it was reported in [10].

3.3. Luminescent Properties. The experimental excitation spectrum for the mixture of the monomer and the dimer is depicted in Figure 6(a). It exhibits a maximum at $355 \mathrm{~nm}$, which can be assigned to the electronic transitions from the ground state level $(\pi) S_{0}$ to the excited level $\left(\pi^{*}\right) S_{1}$ of the cbtfa organic ligand $[14,15,39-41]$, according to Figure 7. The predicted absorption spectra for the gas phase geometries optimized with the Sparkle/PM6 method calculated using the INDO/S-CIS procedure are also shown. Gabedit software 


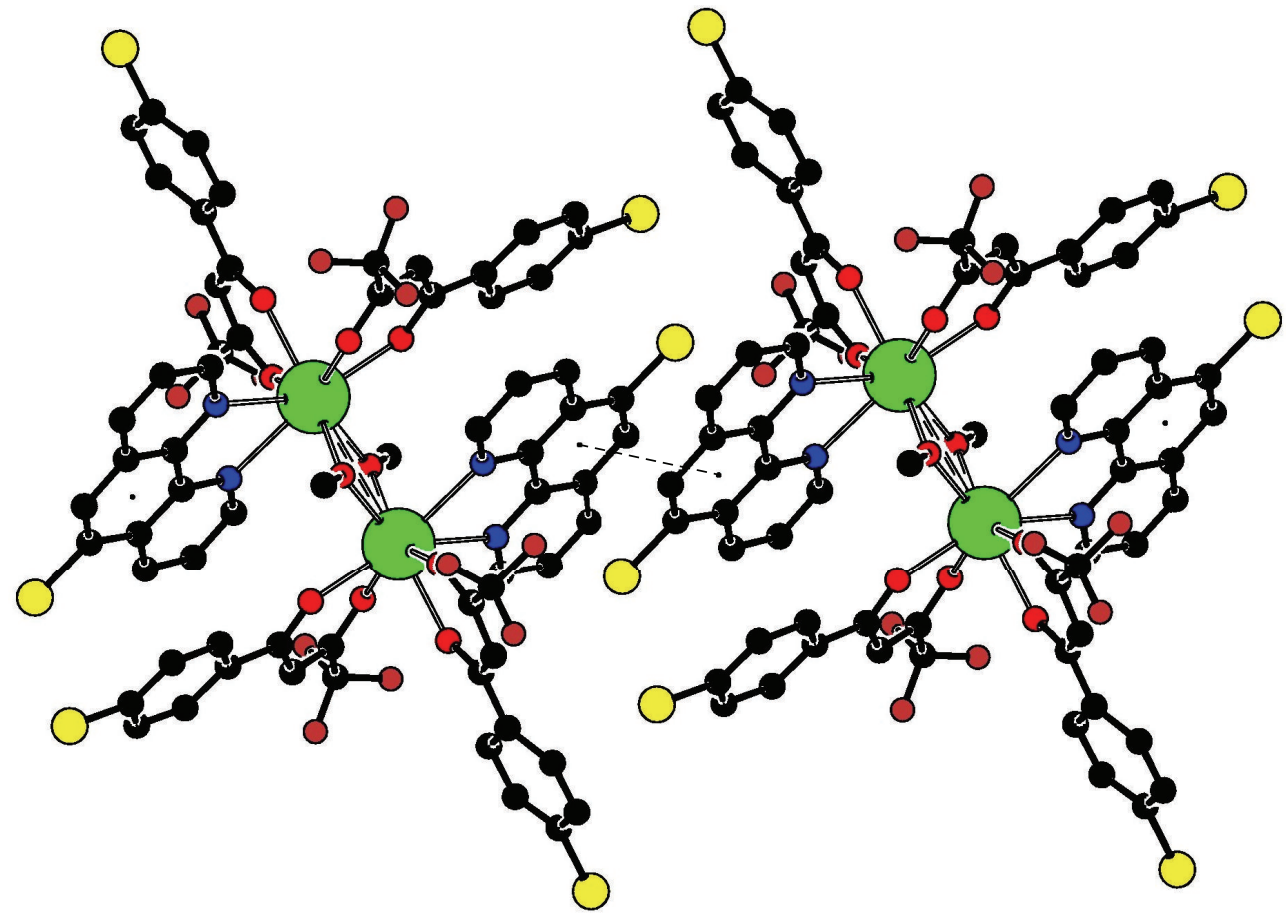

FIgURE 4: Crystal packing of complex 2 , showing $\pi \ldots \pi$ interactions.

TABLE 3: Comparison of the unit cell parameters for PM6 and PM7 predicted structures. In parentheses, for the experimental values, standard deviation is shown; for theoretical values, percent error is indicated.

\begin{tabular}{lcccccc}
\hline Complex & $\begin{array}{c}\text { Complex 1 } \\
\text { SC-XRD }\end{array}$ & $\begin{array}{c}\text { Complex 1 } \\
\text { PM6 model }\end{array}$ & $\begin{array}{c}\text { Complex 1 } \\
\text { PM7 model }\end{array}$ & $\begin{array}{c}\text { Complex 2 } \\
\text { SC-XRD }\end{array}$ & $\begin{array}{c}\text { Complex 2 } \\
\text { PM6 model }\end{array}$ & $\begin{array}{c}\text { Complex 2 } \\
\text { PM7 model }\end{array}$ \\
\hline$a(\AA)$ & $13.246(5)$ & $13.231(0.1 \%)$ & $13.289(0.3 \%)$ & $10.2575(11)$ & $10.876(6.0 \%)$ & $10.408(1.5 \%)$ \\
$b(\AA)$ & $18.252(7)$ & $18.206(0.3 \%)$ & $18.396(0.8 \%)$ & $12.9224(15)$ & $12.648(2.1 \%)$ & $12.722(1.6 \%)$ \\
$c(\AA)$ & $21.081(8)$ & $21.472(1.9 \%)$ & $20.458(3.0 \%)$ & $15.0618(16)$ & $14.901(1.1 \%)$ & $14.420(4.3 \%)$ \\
$\alpha\left({ }^{\circ}\right)$ & $98.241(8)$ & $99.330(1.1 \%)$ & $97.670(0.6 \%)$ & $72.179(6)$ & $69.860(3.2 \%)$ & $72.220(0.1 \%)$ \\
$\beta\left(^{\circ}\right)$ & $99.461(11)$ & $98.860(0.6 \%)$ & $98.770(0.7 \%)$ & $80.460(6)$ & $74.880(6.9 \%)$ & $81.060(0.8 \%)$ \\
$\gamma\left({ }^{\circ}\right)$ & $108.751(10)$ & $108.300(0.4 \%)$ & $108.910(0.2 \%)$ & $84.813(6)$ & $76.650(9.6 \%)$ & $86.170(1.6 \%)$ \\
Volume $\left(\AA^{3}\right)$ & $4654.0(3)$ & $4728.6(1.6 \%)$ & $4585.5(1.5 \%)$ & $1872.7(4)$ & $1835.2(2.0 \%)$ & $1795.7(4.1 \%)$ \\
\hline
\end{tabular}

TABLE 4: Comparison of the experimental and calculated average Eu-N and Eu-O distances (in $\AA$ ) and selected angles (in ${ }^{\circ}$ ) for the mononuclear (monomer 1) and the homodinuclear $\mathrm{Eu}^{3+}$ complexes. In parentheses, for the experimental values, standard deviation is shown; for theoretical values, percent error is indicated.

\begin{tabular}{lcccccc}
\hline \multirow{2}{*}{ Bond distance/angle } & Complex 1 & Complex 1 & Complex 1 & Complex 2 & Complex 2 & Complex 2 \\
& SC-XRD & PM6 model & PM7 model & SC-XRD & PM6 model & PM7 model \\
\hline Eu-O avg. (cbtfa) & $2.371(1)$ & $2.426(2.3 \%)$ & $2.401(1.2 \%)$ & $2.40(2)$ & $2.43(1.4 \%)$ & $2.41(0.4 \%)$ \\
Eu-O avg. (methanol) & - & - & - & 2.301 & $2.412(4.8 \%)$ & $2.318(0.7 \%)$ \\
Eu-N avg. & $2.525(1)$ & $2.497(1.1 \%)$ & $2.545(0.8 \%)$ & $2.641(1)$ & $2.503(5.2 \%)$ & $2.555(3.2 \%)$ \\
\hline O-Eu-O (cbtfa) & $70(1)$ & $62(11.4 \%)$ & $64.17(8.9 \%)$ & $70.4(5)$ & $62.2(11.6 \%)$ & $64.2(8.8 \%)$ \\
O-Eu-O (methanol) & - & - & - & $70.86(1)$ & $74.23(4.8 \%)$ & $73.83(4.2 \%)$ \\
N-Eu-N & $63.3(6)$ & $66.0(4.3 \%)$ & $65.04(2.8 \%)$ & $61.740(6)$ & $65.933(6.8 \%)$ & $64.695(4.8 \%)$ \\
Eu-O-Eu & - & - & - & $109.140(8)$ & $105.767(3.1 \%)$ & $106.172(2.7 \%)$ \\
\hline
\end{tabular}


TABLE 5: Comparison of theoretical (for complex 1 and complex 2) and experimental values (for similar Eu ${ }^{3+}$ complexes reported in the literature) of the intensity parameters $\left(\Omega_{\lambda}\right)$, radiative $\left(A_{\text {rad }}\right)$ and nonradiative $\left(A_{\text {nrad }}\right)$ emission rates, and quantum yields $(\eta)$.

\begin{tabular}{|c|c|c|c|c|c|c|c|c|}
\hline \multirow{2}{*}{ Compound } & \multicolumn{3}{|c|}{ Intensity parameters $\left(10^{-20} \mathrm{~cm}^{2}\right)$} & \multirow{2}{*}{$A_{\text {rad }}\left(\mathrm{s}^{-1}\right)$} & \multirow{2}{*}{$A_{\text {nrad }}\left(\mathrm{s}^{-1}\right)$} & \multirow{2}{*}{$\tau(\mu \mathrm{s})$} & \multirow{2}{*}{$\eta(\%)$} & \multirow{2}{*}{ References } \\
\hline & $\Omega_{2}$ & $\Omega_{4}$ & $\Omega_{6}$ & & & & & \\
\hline Complex 1 & 20.41 & 7.87 & 0.35 & 785.65 & 642.93 & 700 & 54.05 & - \\
\hline Complex 2 (Eul) & 20.40 & 7.90 & 0.26 & 785.73 & 642.84 & 700 & 53.29 & - \\
\hline Complex 2 (Eula) & 20.40 & 7.90 & 0.40 & 785.84 & 642.73 & 700 & 52.87 & - \\
\hline$\left[\mathrm{Eu}(\mathrm{btfa})_{3}(\mathrm{phenNO})\right]$ & 18.6 & - & - & 830 & 650 & 670 & 66 & {$[15]$} \\
\hline$\left[\mathrm{Eu}(\mathrm{btfa})_{3}\left(4,4^{\prime}\right.\right.$-bipy $\left.)(\mathrm{EtOH})\right]$ & 28.8 & 6.7 & - & 1033 & 1189 & 450 & 46.5 & {$[16]$} \\
\hline$\left[\mathrm{Eu}(\mathrm{btfa})_{3}(\right.$ phen $\left.)\right]$ & - & - & - & 580 & 569 & 210 & 50 & {$[17]$} \\
\hline$\left[\mathrm{Eu}(\mathrm{tta})_{3}(\mathrm{phen})\right]$ & - & - & - & 436 & 993 & 700 & 31 & [17] \\
\hline$\left[\mathrm{Eu}(\mathrm{NTA})_{3}(\right.$ phen $\left.)\right]$ & - & - & - & 600 & 900 & 662 & 40 & [17] \\
\hline
\end{tabular}

$\mathrm{btfa}=$ 4,4,4-trifluoro-1-phenyl-1,3-butanedionate; $\mathrm{tta}=2$-thenoyltrifluoroacetonate; NTA = 1-(2-naphthoyl)-3,3,3-trifluroacetonate; phen = phenanthroline; phenNO = 1,10-phenanthroline-N-oxide; $4,4^{\prime}$-bipy $=4,4^{\prime}$-bipyridine.

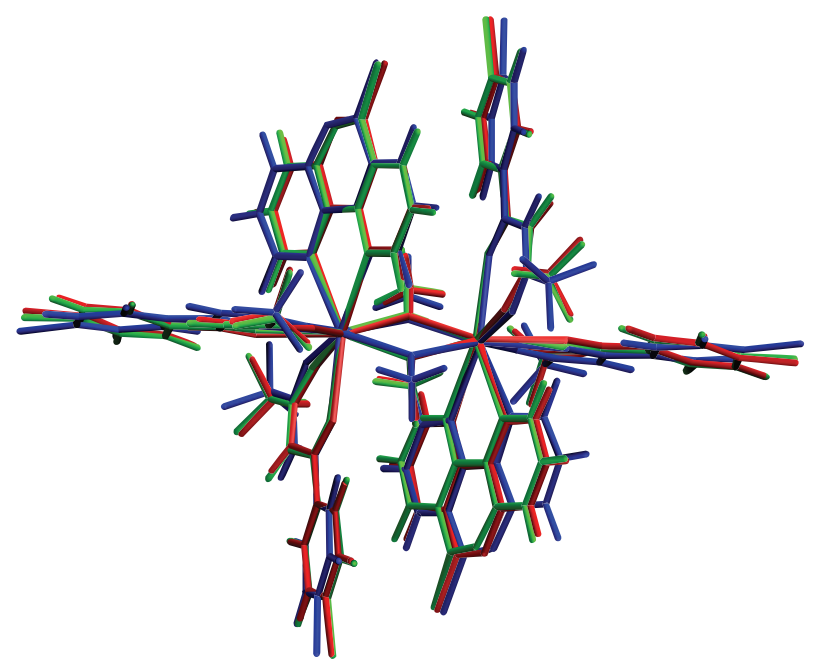

FIgure 5: Comparison of the Sparkle/PM6 (blue) and Sparkle/PM7 (green) optimized geometries with the X-ray geometry (red) of complex 2 (software used for visualization: VMD version 1.9.1 [38]).

[42] has been used for the representation of the spectra from the calculated data. As expected, there is a hypsochromic shift for the lowest predicted transition (versus the experimental one), as it is usually the case in this type of calculations [3].

Upon excitation of the organic ligands in the UV region, efficient indirect excitation of $\mathrm{Eu}^{3+}$ is attained, via antenna effect [43]. Figure 6(b) shows the experimental photoluminescence spectrum, in which the characteristic narrow emission bands of $\mathrm{Eu}^{3+}$ corresponding to the intraconfigurational ${ }^{5} \mathrm{D}_{0} \rightarrow{ }^{7} \mathrm{~F}_{J}(J=0-4)$ transitions appear. The five expected peaks for the ${ }^{5} \mathrm{D}_{0} \rightarrow{ }^{7} \mathrm{~F}_{0-4}$ transitions (namely, ${ }^{5} \mathrm{D}_{0} \rightarrow{ }^{7} \mathrm{~F}_{0}$ $(\sim 580 \mathrm{~nm}),{ }^{5} \mathrm{D}_{0} \rightarrow{ }^{7} \mathrm{~F}_{1}(\sim 591 \mathrm{~nm}),{ }^{5} \mathrm{D}_{0} \rightarrow{ }^{7} \mathrm{~F}_{2}(\sim 614 \mathrm{~nm})$, ${ }^{5} \mathrm{D}_{0} \rightarrow{ }^{7} \mathrm{~F}_{3}(\sim 651 \mathrm{~nm})$, and $\left.{ }^{5} \mathrm{D}_{0} \rightarrow{ }^{7} \mathrm{~F}_{4}(\sim 692 \mathrm{~nm})\right)$ [44] can be identified (see Figure 7 ).

The emission bands at ca. 580 and $651 \mathrm{~nm}$ are weak since their corresponding transitions ${ }^{5} \mathrm{D}_{0} \rightarrow{ }^{7} \mathrm{~F}_{0,3}$ are forbidden both in magnetic and electric dipole schemes [47]. The intensity of the emission band at $593 \mathrm{~nm}$ is stronger and independent of the coordination environment because the corresponding transition ${ }^{5} \mathrm{D}_{0} \rightarrow{ }^{7} \mathrm{~F}_{1}$ is of magnetic character. In contrast, the ${ }^{5} \mathrm{D}_{0} \rightarrow{ }^{7} \mathrm{~F}_{2}$ transition is an induced electric dipole transition and its corresponding intense emission at $613 \mathrm{~nm}$ is very sensitive to the coordination environment [47]. This very intense ${ }^{5} \mathrm{D}_{0} \rightarrow{ }^{7} \mathrm{~F}_{2}$ peak, responsible for the brilliant red emission of the complex, indicates that the ligand field surrounding the $\mathrm{Eu}^{3+}$ ion is highly polarizable.

With regard to the monochromaticity $(R)$, that is, the intensity ratio of the electric dipole transition to the magnetic dipole transition (red/orange ratio), the obtained value is 17.2. This indicates that the CIE chromaticity coordinates for the complex should be very close to saturated red emission [48] and that the $\mathrm{Eu}^{3+}$ coordination is consistent with a local site without inversion [49-51].

The lifetime measurements for the mixture of the monomer and the dimer (not shown) do not correspond to a monoexponential decay curve, which is consistent with the presence of two slightly different coordination environments for the $\mathrm{Eu}^{3+}$ ion in complex 1 and complex 2. The estimated average $\tau$ value would be close to $700 \mu \mathrm{s}$, in the same range as the lifetimes reported for similar complexes with cbtfa and btfa ligands: $754 \mu \mathrm{s}$ for [Eu(cbtfa) ${ }_{3}$ (bath)] [14], $670 \mu \mathrm{s}$ for $\left[\operatorname{Er}(\mathrm{btfa})_{3}(\mathrm{phen})\right][15,52]$, and $750 \mu \mathrm{s}\left[\mathrm{Eu}(\mathrm{btfac})_{3}\right.$ (dmbipy) [53].

3.4. Theoretical Modeling of the Luminescent Properties with LUMPAC. By using LUMPAC lanthanide luminescence software package [11-13], singlet and triplet excited state energies for the lanthanide containing systems were obtained from INDO/S-CIS ORCA [27] calculations. From the experimental emission spectrum and estimated lifetime value, Judd-Ofelt intensity parameters, radiative and nonradiative emission rates, and quantum efficiencies were also calculated. The isolated complex ground state geometries have been used in the calculations. As discussed above, the two $\mathrm{Eu}^{3+}$ ions in complex 2 have been treated individually and are described as Eul and Eula in Table 5.

The estimated singlet $\left(36827.40 \mathrm{~cm}^{-1}\right.$ for the monomer and $36945.60 \mathrm{~cm}^{-1}$ for the dimer) is higher than that of the cbtfa ligand $\left(30675 \mathrm{~cm}^{-1}\right.$ [16]) but would be a reasonable 


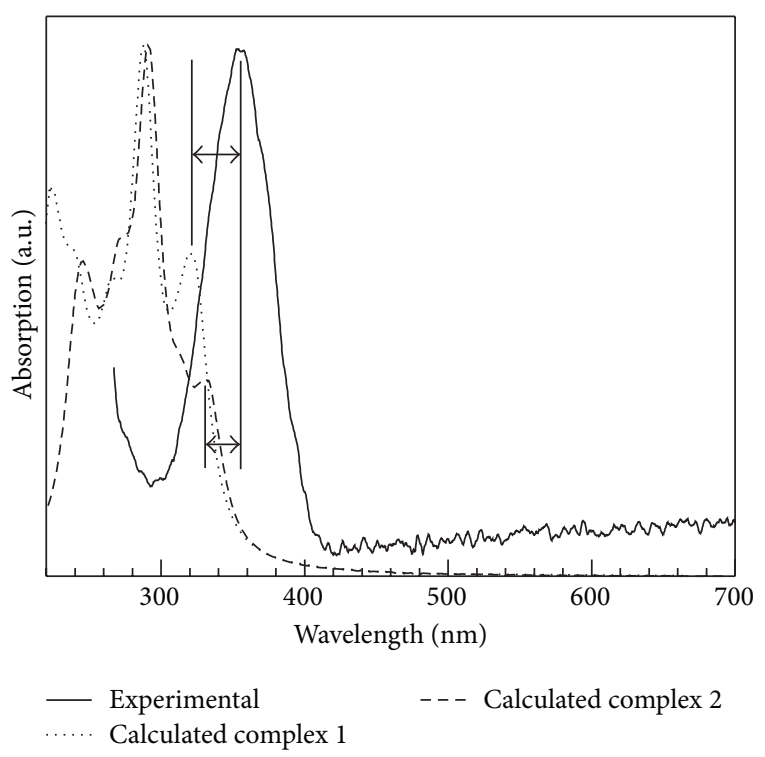

(a)

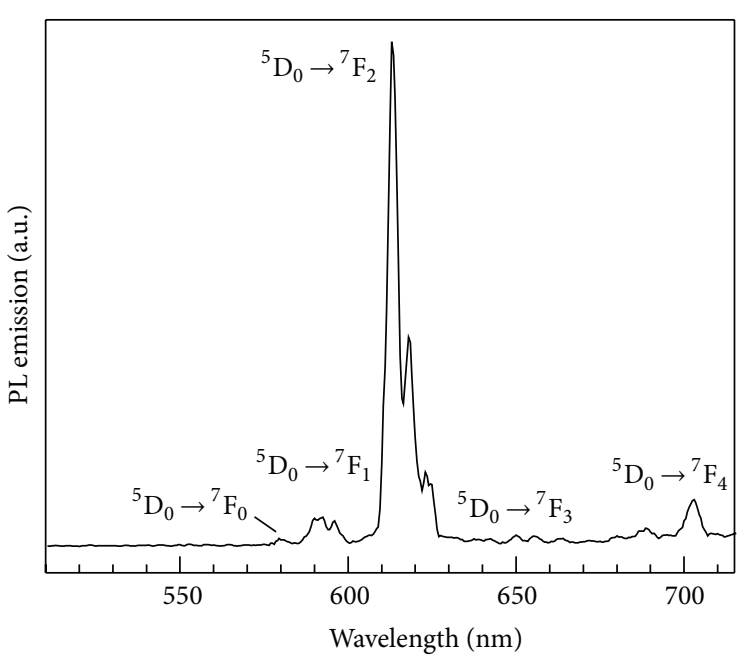

(b)

FIGURE 6: (a) UV-Vis absorption spectrum and (b) photoluminescent emission spectrum in powder form for the mixture of complex 1 and complex 2 upon ligand-mediated excitation in the UV $(\lambda=355 \mathrm{~nm})$.

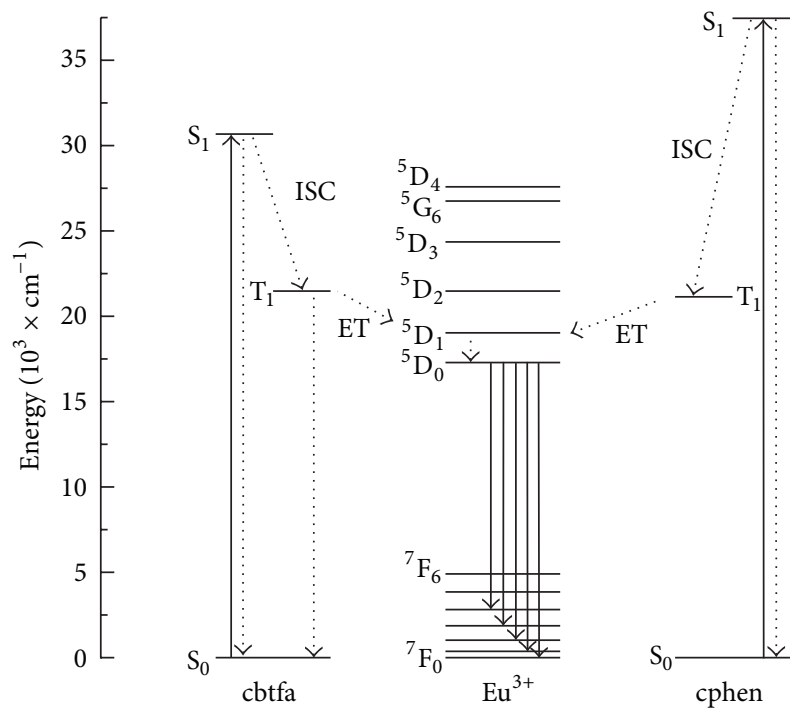

Figure 7: Scheme of the energy transfer mechanism and emission process. The singlet and triplet values of Hcbtfa have been estimated from those reported for $\mathrm{Hbtfa}[16,45]$. The singlet and triplet values for 5-chloro-1,10-phenanthroline have been taken from [46]. ISC and ET stand for intersystem crossing and energy transfer, respectively.

estimation of the singlet of 5-chloro-1,10-phenanthroline $\left(37453 \mathrm{~cm}^{-1}\right.$ [46]). Figure 8(a) shows the lowest unoccupied molecular orbital (LUMO) of complex 1 plotted using Gabedit software [42] with the data from MOPAC calculations and Figures 8(b) and 8(c) depict the two lowest (almost degenerate) unoccupied molecular orbitals of complex 2, corroborating that, in all cases, they correspond to cphen diimide.
Regarding the triplet position (estimated in $20040.60 \mathrm{~cm}^{-1}$ for complex 1 and in $20172.50 \mathrm{~cm}^{-1}$ for complex 2), it is close to that of cphen $\left(21142 \pm 45 \mathrm{~cm}^{-1}\right.$ [46]) and to that of the cbtfa ligand $\left(20276 \mathrm{~cm}^{-1}[16]\right.$ or $21.277 \mathrm{~cm}^{-1}$ [45]), so it is not possible to discern whether it corresponds to the $\beta$-diketonate and to the $\mathrm{N}, \mathrm{N}$-donor or if both would play a role in the energy transfer to the $\mathrm{Eu}^{3+}$ ion (the most usual situation). For $\mathrm{Eu}^{3+}$ complexes with dithiocarbamate and different N,N-donors (including cphen), Regulacio et al. [46] suggested that the intramolecular energy transfer to the emissive states of the lanthanide was predominantly from the triplet state of the bidentate aromatic amine and that there may be intramolecular energy migration from the dithiocarbamate to the bidentate aromatic ligand.

In relation to the Judd-Ofelt intensity parameters, $\Omega_{\lambda}$ $(\lambda=2,4$, and 6$)$, they are summarized in Table 5. It is worth noting that, for the most similar complex (i.e., [Eu(btfa $)_{3}\left(\right.$ phenNO)]), the estimated $\Omega_{2}$ value is pretty close, and so are the radiative and nonradiative decay rates, so the software provides a good estimation. For other $\mathrm{Eu}^{3+}$ complexes with different fluorinated $\beta$-diketonates, the differences in the estimated values are, as expected, more evident.

\section{Conclusions}

Taking a $\beta$-diketone which is known to optimize the quantum yield of $\mathrm{Eu}^{3+}$ complexes, 1-(4-chlorophenyl)-4,4,4trifluoro-1,3-butanedione (Hcbtfa), and neutral diimide, 5chloro-1,10-phenanthroline (cphen), as coordinating ligands, two octacoordinated complexes were synthesized: a monomer, formulated as $\left[\mathrm{Eu}(\mathrm{cbtfa})_{3}(\mathrm{cphen})\right]$, and a dimer, $\left[\mathrm{Eu}_{2}(\mathrm{cbtfa})_{4}(\mathrm{cphen})_{2}\left(\mathrm{CH}_{3} \mathrm{O}\right)_{2}\right]$. The experimental 


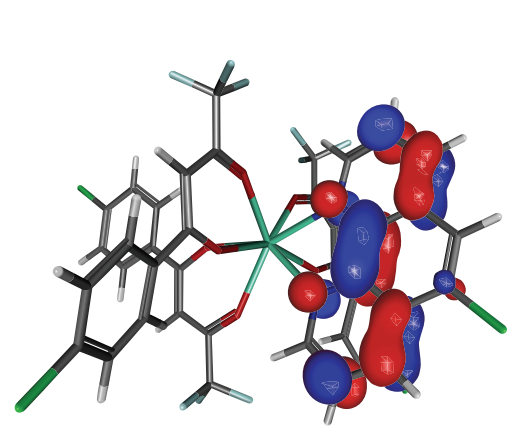

(a)

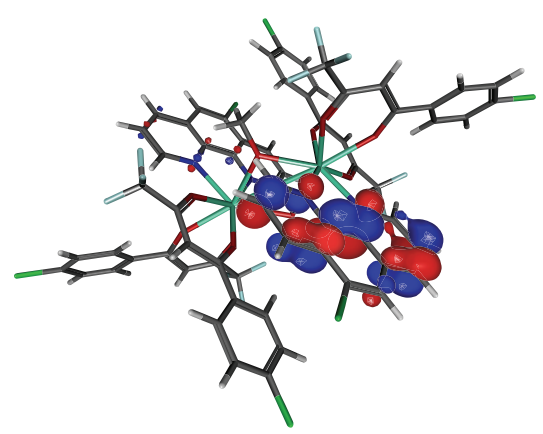

(b)

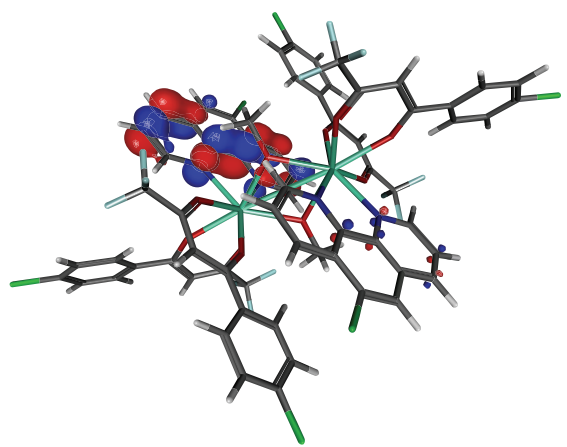

(c)

Figure 8: LUMO level for complex 1 (a) and for complex 2 (b and c).

characterization data (X-ray structural elucidation, UV-Vis absorption, and PL emission) for a mixture of these two complexes was subsequently used so as to assess a recently released quantum chemistry software: LUMPAC.

The predicted equilibrium energy configurations calculated by semiempirical methods (Sparkle/PM6 and Sparkle/PM7 Hamiltonians) showed percent errors below $5 \%$ in all cases (and generally below 2\%) for the unit cell parameters (versus X-ray structures), thus providing a good estimation. The same applied to the distances and angles for the first coordination sphere (although in this case Sparkle/PM7 Hamiltonian attained a significantly better accuracy than PM6).

In relation to LUMPAC's second module, the estimations of the singlet and triplet obtained by INDO/S-CIS method were remarkably good, and the calculated UV-Vis absorption spectra could be regarded as an acceptable approximation for practical purposes.

Regarding the third module, the Judd-Ofelt intensity parameters, radiative and nonradiative emission rates, and quantum efficiencies appeared to be in good agreement with those of similar complexes reported in the literature, although further research needs to be conducted to confirm these preliminary findings.

All considered, LUMPAC software can be deemed as a very promising tool for the design of novel $\operatorname{Ln}(\mathrm{III})$ complexes, given its computational efficiency and ease of use, in addition to being free of charge.

\section{Conflict of Interests}

The authors declare that there is no conflict of interests regarding the publication of this paper.

\section{Acknowledgments}

Pablo Martín-Ramos would like to gratefully acknowledge the financial support of Santander Universidades through "Becas Iberoamérica Jóvenes Profesores e Investigadores, España 2015" scholarship program. The authors also wish to thank Professor I. R. Martín and Professor V. Lavín (Universidad de La Laguna) for their insightful discussions.

\section{References}

[1] J.-C. G. Bünzli, S. Comby, A.-S. Chauvin, and C. D. B. Vandevyver, "New opportunities for lanthanide luminescence," Journal of Rare Earths, vol. 25, no. 3, pp. 257-274, 2007.

[2] S. Comby and J.-C. G. Bünzli, "Lanthanide near-infrared luminescence in molecular probes and devices," in Optical Spectroscopy, V. K. Pecharsky, J. C. G. Bünzli, and K. A. Gschneider Jr., Eds., pp. 217-470, Elsevier, 2007.

[3] G. F. de Sá, O. L. Malta, C. de Mello Donegá et al., "Spectroscopic properties and design of highly luminescent lanthanide coordination complexes," Coordination Chemistry Reviews, vol. 196, no. 1, pp. 165-195, 2000.

[4] R. O. Freire, R. Q. Albuquerque, S. A. Junior, G. B. Rocha, and M. E. de Mesquita, "On the use of combinatory chemistry to the design of new luminescent Eu ${ }^{3+}$ complexes," Chemical Physics Letters, vol. 405, no. 1-3, pp. 123-126, 2005.

[5] R. O. Freire, F. R. G. Silva, M. O. Rodrigues, M. E. de Mesquita, and N. B. da Costa Jr., "Design of europium(III) complexes with high quantum yield," Journal of Molecular Modeling, vol. 12, no. 1, pp. 16-23, 2005.

[6] J. D. L. Dutra, I. F. Gimenez, N. B. D. C. Junior, and R. O. Freire, "Theoretical design of highly luminescent europium (III) complexes: a factorial study," Journal of Photochemistry and Photobiology A: Chemistry, vol. 217, no. 2-3, pp. 389-394, 2011.

[7] N. B. D. Lima, S. M. C. Gonçalves, S. A. Júnior, and A. M. Simas, "A comprehensive strategy to boost the quantum yield of luminescence of europium complexes," Scientific Reports, vol. 3, article 2395, 2013.

[8] R. O. Freire, G. B. Rocha, R. Q. Albuquerque, and A. M. Simas, "Efficacy of the semiempirical sparkle model as compared to ECP ab-initio calculations for the prediction of ligand field parameters of europium (III) complexes," Journal of Luminescence, vol. 111, no. 1-2, pp. 81-87, 2005.

[9] D. A. Rodrigues, N. B. da Costa Jr., and R. O. Freire, "Would the pseudocoordination centre method be appropriate to describe the geometries of lanthanide complexes?" Journal of Chemical Information and Modeling, vol. 51, no. 1, pp. 45-51, 2011.

[10] P. Martín-Ramos, P. S. P. Silva, P. Chamorro-Posada et al., "Synthesis, structure, theoretical studies and luminescent properties of a ternary erbium(III) complex with acetylacetone and bathophenanthroline ligands," Journal of Luminescence, vol. 162, pp. 41-49, 2015.

[11] J. D. L. Dutra, T. D. Bispo, and R. O. Freire, "LUMPAC lanthanide luminescence software: efficient and user friendly," 
Journal of Computational Chemistry, vol. 35, no. 10, pp. 772-775, 2014.

[12] J. D. L. Dutra and R. O. Freire, "Theoretical tools for the calculation of the photoluminescent properties of europium systemsa case study," Journal of Photochemistry and Photobiology A: Chemistry, vol. 256, pp. 29-35, 2013.

[13] J. D. L. Dutra, J. W. Ferreira, M. O. Rodrigues, and R. O. Freire, "Theoretical methodologies for calculation of Judd-Ofelt intensity parameters of polyeuropium systems," The Journal of Physical Chemistry A, vol. 117, no. 51, pp. 14095-14099, 2013.

[14] J. P. Martins, P. Martín-Ramos, C. Coya et al., "Highly luminescent pure-red-emitting fluorinated $\beta$-diketonate europium(III) complex for full solution-processed OLEDs," Journal of Luminescence, vol. 159, pp. 17-25, 2015.

[15] C. de Mello Donegá, S. Alves Jr., and G. F. de Sá, "Synthesis, luminescence and quantum yields of $\mathrm{Eu}(\mathrm{III})$ mixed complexes with 4,4,4-trifluoro-1-phenyl-1,3-butanedione and 1,10phenanthroline-N-oxide," Journal of Alloys and Compounds, vol. 250, no. 1-2, pp. 422-426, 1997.

[16] P. P. Lima, R. A. Sá Ferreira, R. O. Freire et al., "Spectroscopic study of a UV-photostable organic-inorganic hybrids incorporating an $\mathrm{Eu}^{3+} \beta$-diketonate complex," ChemPhysChem, vol. 7, no. 3, pp. 735-746, 2006.

[17] S. Biju, D. B. A. Raj, M. L. P. Reddy, and B. M. Kariuki, "Synthesis, crystal structure, and luminescent properties of novel $\mathrm{Eu}^{3+}$ heterocyclic $\beta$-diketonate complexes with bidentate nitrogen donors," Inorganic Chemistry, vol. 45, no. 26, pp. 1065110660, 2006.

[18] G. Sheldrick, SADABS, University of Göttingen, Göttingen, Germany, 1996.

[19] G. M. Sheldrick, "A short history of SHELX," Acta Crystallographica Section A: Foundations of Crystallography, vol. 64, no. 1, pp. 112-122, 2007.

[20] A. L. Spek, "Single-crystal structure validation with the program PLATON," Journal of Applied Crystallography, vol. 36, no. 1, pp. 7-13, 2003.

[21] J. J. P. Stewart, "Optimization of parameters for semiempirical methods V: modification of NDDO approximations and application to 70 elements," Journal of Molecular Modeling, vol. 13, no. 12, pp. 1173-1213, 2007.

[22] R. O. Freire and A. M. Simas, "Sparkle/PM6 parameters for all lanthanide trications from $\mathrm{La}(\mathrm{III})$ to $\mathrm{Lu}(\mathrm{III})$," Journal of Chemical Theory and Computation, vol. 6, no. 7, pp. 2019-2023, 2010.

[23] J. J. P. Stewart, "Optimization of parameters for semiempirical methods VI: more modifications to the NDDO approximations and re-optimization of parameters," Journal of Molecular Modeling, vol. 19, no. 1, pp. 1-32, 2013.

[24] J. D. L. Dutra, M. A. M. Filho, G. B. Rocha, R. O. Freire, A. M. Simas, and J. J. P. Stewart, "Sparkle/PM7 lanthanide parameters for the modeling of complexes and materials," Journal of Chemical Theory and Computation, vol. 9, no. 8, pp. 3333-3341, 2013.

[25] J. J. P. Stewart, MOPAC2012, Stewart Computational Chemistry, Colorado Springs, Colo, USA, 2012.

[26] J. D. C. Maia, G. A. Urquiza Carvalho, C. P. Mangueira Jr., S. R. Santana, L. A. F. Cabral, and G. B. Rocha, "GPU linear algebra libraries and GPGPU programming for accelerating MOPAC semiempirical quantum chemistry calculations," Journal of Chemical Theory and Computation, vol. 8, no. 9, pp. 3072-3081, 2012.
[27] F. Neese, “The ORCA program system," Wiley Interdisciplinary Reviews: Computational Molecular Science, vol. 2, no. 1, pp. 7378, 2012.

[28] T. Petrenko and F. Neese, "Analysis and prediction of absorption band shapes, fluorescence band shapes, resonance Raman intensities, and excitation profiles using the time-dependent theory of electronic spectroscopy," Journal of Chemical Physics, vol. 127, no. 16, Article ID 164319, 2007.

[29] J. Ridley and M. Zerner, "An intermediate neglect of differential overlap technique for spectroscopy: pyrrole and the azines," Theoretica Chimica Acta, vol. 32, no. 2, pp. 111-134, 1973.

[30] M. C. Zerner, G. H. Loew, R. F. Kirchner, and U. T. MuellerWesterhoff, "An intermediate neglect of differential overlap technique for spectroscopy of transition-metal complexes. Ferrocene," Journal of the American Chemical Society, vol. 102, no. 2, pp. 589-599, 1980.

[31] J. E. Ridley and M. C. Zerner, "Triplet states via intermediate neglect of differential overlap: benzene, pyridine and the diazines," Theoretica Chimica Acta, vol. 42, no. 3, pp. 223-236, 1976.

[32] A. V. M. de Andrade, R. L. Longo, A. M. Simas, and G. F. de Sá, "Theoretical model for the prediction of electronic spectra of lanthanide complexes," Journal of the Chemical Society: Faraday Transactions, vol. 92, no. 11, pp. 1835-1839, 1996.

[33] A. V. M. de Andrade, N. B. da Costa Jr., O. L. Malta, R. L. Longo, A. M. Simas, and G. F. de Sá, "Excited state calculations of Europium(III) complexes," Journal of Alloys and Compounds, vol. 250, no. 1, pp. 412-416, 1997.

[34] O. L. Malta, M. A. C. dos Santos, L. C. Thompson, and N. K. Ito, "Intensity parameters of $4 \mathrm{f}-4 \mathrm{f}$ transitions in the Eu(dipivaloylmethanate)3 1, 10-phenanthroline complex," Journal of Luminescence, vol. 69, no. 2, pp. 77-84, 1996.

[35] O. L. Malta, "Mechanisms of non-radiative energy transfer involving lanthanide ions revisited," Journal of Non-Crystalline Solids, vol. 354, no. 42-44, pp. 4770-4776, 2008.

[36] O. L. Malta, H. F. Brito, J. F. S. Menezes, F. R. Gonçalves e Silva, C. de Mello Donegá, and S. Alves Jr., "Experimental and theoretical emission quantum yield in the compound Eu(thenoyltrifluoroacetonate)3.2(dibenzyl sulfoxide)," Chemical Physics Letters, vol. 282, no. 3-4, pp. 233-238, 1998.

[37] A. G. Orpen, L. Brammer, F. H. Allen, O. Kennard, D. G. Watson, and R. Taylor, "Supplement. Tables of bond lengths determined by X-ray and neutron diffraction. Part 2. Organometallic compounds and co-ordination complexes of the d-and f-block metals," Journal of the Chemical Society, Dalton Transactions, no. 12, pp. S1-S83, 1989.

[38] W. Humphrey, A. Dalke, and K. Schulten, "VMD: visual molecular dynamics," Journal of Molecular Graphics, vol. 14, no. 1, pp. 33-38, 1996.

[39] J.-F. Guo, H.-J. Zhang, L.-S. Fu, and Q.-G. Meng, "Crystal structure and luminescent properties of the complex [Eu(III)(TFPB $)_{3}$ bpy]," Chinese Journal of Inorganic Chemistry, vol. 20, no. 5, pp. 543-546, 2004.

[40] M. T. Mongelli and K. J. Brewer, "Synthesis and study of the light absorbing, redox and photophysical properties of $\mathrm{Ru}(\mathrm{II})$ and Os(II) complexes of 4,7-diphenyl-1,10-phenanthroline containing the polyazine bridging ligand 2,3-bis(2-pyridyl)pyrazine," Inorganic Chemistry Communications, vol. 9, no. 9, pp. 877-881, 2006.

[41] M. A. Ivanov, M. V. Puzyk, T. A. Tkacheva, and K. P. Balashev, "Effect of heterocyclic diimine ligands with donor and acceptor 
substituents on the spectroscopic and electrochemical properties of Au(III) complexes," Russian Journal of General Chemistry, vol. 76, no. 2, pp. 165-169, 2006.

[42] A.-R. Allouche, "Gabedita-a graphical user interface for computational chemistry softwares," Journal of Computational Chemistry, vol. 32, no. 1, pp. 174-182, 2011.

[43] J.-M. Lehn, "Perspectives in supramolecular chemistry-from molecular recognition towards molecular information processing and self-organization," Angewandte Chemie, vol. 29, no. 11, pp. 1304-1319, 1990.

[44] C. Görller-Walrand and K. Binnemans, "Spectral intensities of f-f transitions," in Handbook on the Physics and Chemistry of Rare Earths, K. A. Gschneidner and L. Eyring, Eds., pp. 101-264, Elsevier, Amsterdam, The Netherlands, 1998.

[45] E. Stathatos, P. Lianos, E. Evgeniou, and A. D. Keramidas, "Electroluminescence by a $\mathrm{Sm}^{3+}$-diketonate-phenanthroline complex," Synthetic Metals, vol. 139, no. 2, pp. 433-437, 2003.

[46] M. D. Regulacio, M. H. Pablico, J. A. Vasquez et al., "Luminescence of $\mathrm{Ln}$ (III) dithiocarbamate complexes $(\mathrm{Ln}=\mathrm{La}, \mathrm{Pr}, \mathrm{Sm}$, $\mathrm{Eu}, \mathrm{Gd}, \mathrm{Tb}, \mathrm{Dy})$," Inorganic Chemistry, vol. 47, no. 5, pp. 15121523, 2008.

[47] M. H. V. Werts, R. T. F. Jukes, and J. W. Verhoeven, “The emission spectrum and the radiative lifetime of $\mathrm{Eu}^{3+}$ in luminescent lanthanide complexes," Physical Chemistry Chemical Physics, vol. 4, no. 9, pp. 1542-1548, 2002.

[48] V. Divya, S. Biju, R. L. Varma, and M. L. P. Reddy, "Highly efficient visible light sensitized red emission from europium tris[1-(4-biphenoyl)-3-(2-fluoroyl)propanedione](1,10-phenanthroline) complex grafted on silica nanoparticles," Journal of Materials Chemistry, vol. 20, no. 25, pp. 5220-5227, 2010.

[49] S. Biju, M. L. P. Reddy, A. H. Cowley, and K. V. Vasudevan, "3-Phenyl-4-acyl-5-isoxazolonate complex of $\mathrm{Tb}^{3+}$ doped into poly- $\beta$-hydroxybutyrate matrix as a promising light-conversion molecular device," Journal of Materials Chemistry, vol. 19, no. 29, pp. 5179-5187, 2009.

[50] A. F. Kirby, D. Foster, and F. S. Richardson, "Comparison of ${ }^{7} \mathrm{~F}_{J} \leftarrow{ }^{5} \mathrm{D}_{\mathrm{O}}$ emission spectra for $\mathrm{Eu}(\mathrm{III})$ in crystalline environments of octahedral, near-octahedral, and trigonal symmetry," Chemical Physics Letters, vol. 95, no. 6, pp. 507-512, 1983.

[51] G. Ligner, R. Mohan, S. Knittel, and G. Duportail, "Hypersensitivity of terbium and europium ions luminescence in biological substrates," Spectrochimica Acta Part A: Molecular Spectroscopy, vol. 46, no. 5, pp. 797-802, 1990.

[52] C. D. M. Donegá, S. A. Junior, and G. F. de Sá, "Europium(III) mixed complexes with $\beta$-diketones and o-phenanthroline- $N$ oxide as promising light-conversion molecular devices," Chemical Communications, no. 10, pp. 1199-1200, 1996.

[53] C. R. de Silva, J. R. Maeyer, R. Wang, G. S. Nichol, and Z. Zheng, "Adducts of europium $\beta$-diketonates with nitrogen $p, p^{\prime}$ disubstituted bipyridine and phenanthroline ligands: synthesis, structural characterization, and luminescence studies," Inorganica Chimica Acta, vol. 360, no. 11, pp. 3543-3552, 2007. 

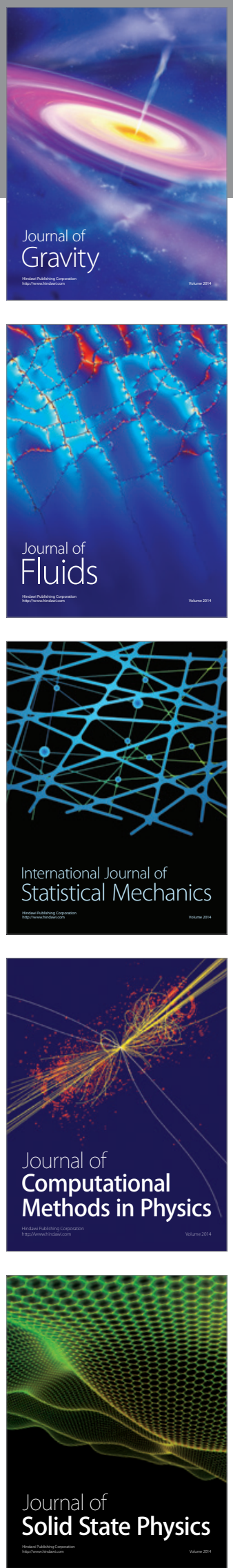

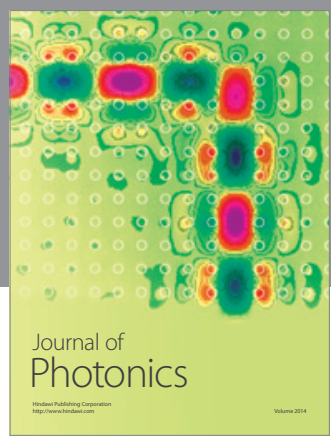

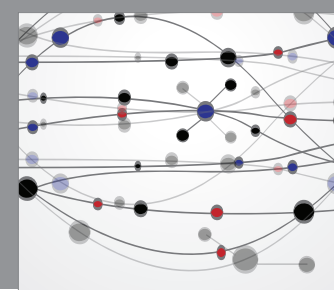

The Scientific World Journal

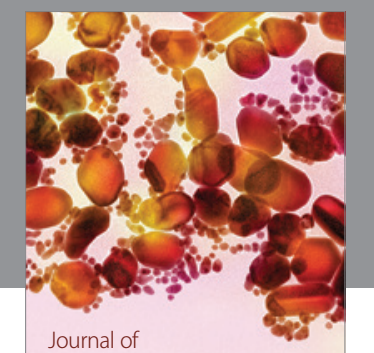

Soft Matter
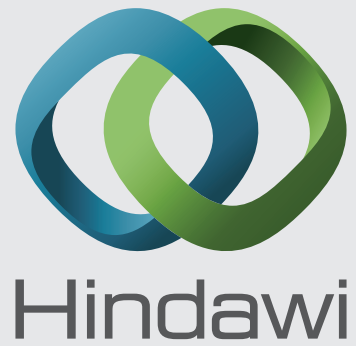

Submit your manuscripts at

http://www.hindawi.com
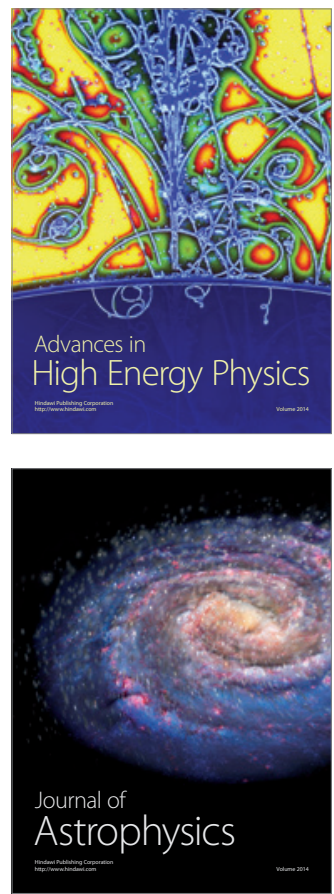
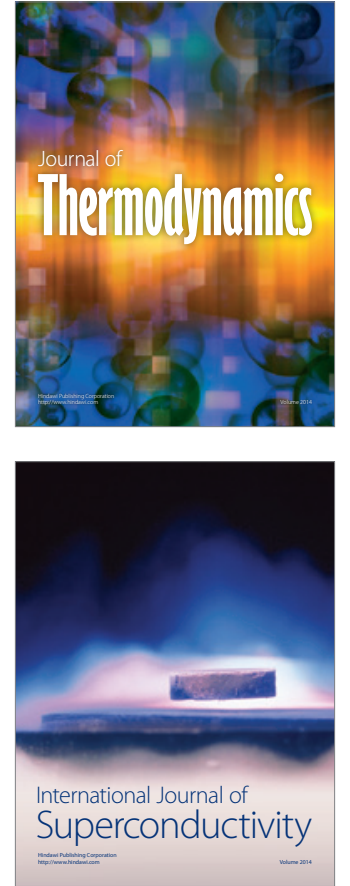
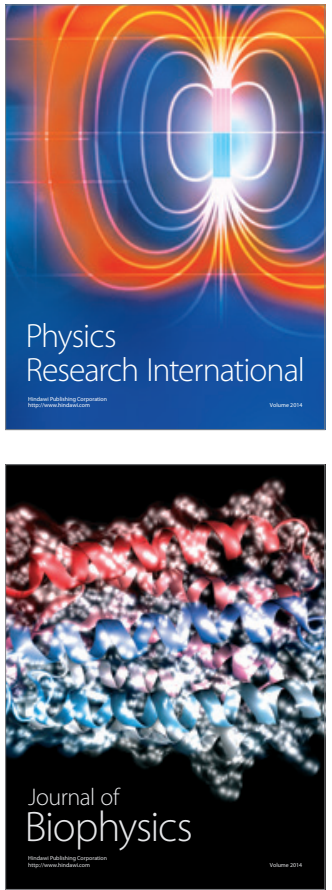
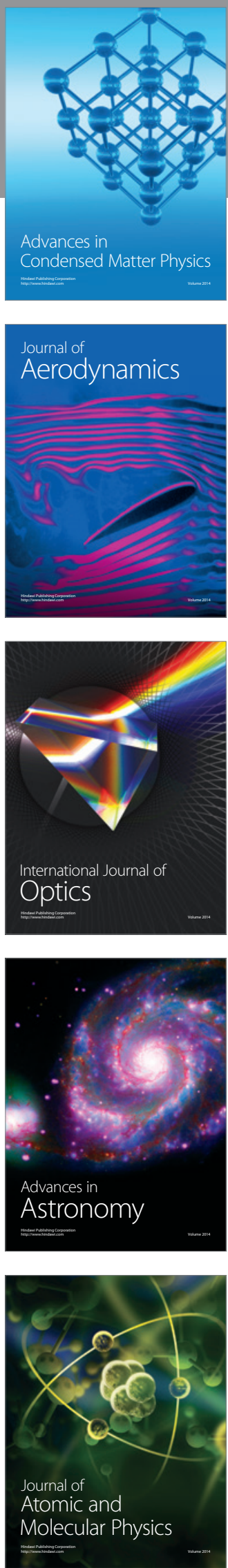